\title{
APLICACIÓN DE UN JUEGO DE EMPRESA COMO RECURSO DIDÁCTICO PARA MEJORAR LA ENSEÑANZA-APRENDIZAJE DEL PLANEAMIENTO Y CONTROL DE LA PRODUCCIÓN SEGÚN EL MODELO DE TEORÍA DE RESTRICCIONES
}

Flor de Maria Tapia Vargas

\section{Resumen}

El objetivo de esta investigación es elaborar un Juego de Empresa, para mejorar la enseñanza-aprendizaje del Planeamiento y Control de la Producción, según el Modelo de Teoría de Restricciones. El Juego es una simulación de una Línea de Producción con ocho centros de trabajo.

Para probar las hipótesis, desarrollamos una investigación experimental, en la Maestría en Ingeniería Industrial de la Escuela de Posgrado de la Universidad Ricardo Palma (Lima- Perú).

Se trabajó con un Grupo de Control y un Grupo Experimental. Para evaluar si el Juego mejora el aprendizaje del Planeamiento y Control de la Producción, el Grupo Experimental trabajó con el Juego, mientras el Grupo de Control utilizó Hoja de Cálculo con números aleatorios para simular la misma Línea de Producción.

Palabras clave: Juego de Empresa; Sistema de Teoría de Restricciones; Flujo de Producción; Tambor-Amortiguador-Cuerda (TAC); Inventario en Proceso.

\section{Abstract}

The objective of this Investigation is to make a business game available as an educational resource for the improvement of the teaching and learning experience of Production Planning and Control, based on the Theory of Constraints System. The Game consists of a simulation of a Production Line with eight work centers. 
An experimental research was developed at the Graduate Program of Industrial Engineering at Universidad Ricardo Palma (Lima - Peru) in order to attain the study objectives and demonstrate our hypothesis.

The study used one control group and one experimental group. As the objective was to assess whether the application of the Game improves learning of Production Planning and Control, the Experimental Group was allowed to work with the Game, while the Control Group used a Spreadsheet with random numbers to simulate the same Production Line.

Keywords: Business Game; Theory of Constraints System; Throughput; Drum-Buffer-Rope (DBR); Work in Process.

\section{INTRODUCCIÓN}

¿Qué pensamiento se dispara en nuestra cabeza cuando hablamos de "Juego de Empresa"? Probablemente, imaginamos una actividad recreativa o de entretenimiento y, quizás, dejamos en segundo plano la connotación didáctica de este término.

Un Juego de Empresa es un método activo de enseñanza-aprendizaje, por medio del cual los jugadores simulan una realidad empresarial, y obtienen, procesan y analizan, de manera lúdica, un conjunto de informaciones que les permiten encontrar soluciones prácticas al problema simulado.

Según Kolb (1997), un Juego de Empresa es una forma de aprendizaje vivencial que hace posible un aprendizaje efectivo. Con los Juegos de Empresa, lo fundamental está en el experimentar y en el aprender haciendo. Según Souza \& Lopes (2004), el pedagogo pasa a asumir nuevos papeles frente a sus pupilos, como orientador, incentivador, facilitador, motivador o consultor.

Ahora bien, en esta investigación estamos proponiendo un Juego de
Empresa para mejorar la enseñanzaaprendizaje del Planeamiento y Control de la Producción, según el Modelo de la Teoría de Restricciones.

Pero, ¿por qué poner a disposición de la comunidad académica y empresarial este recurso?

A continuación, presentamos las respuestas a esta interrogante, las que nos permitirán aproximarnos a una realidad problemática y a la vez justificar nuestro estudio.

- Primero: hay un paradigma enquistado en el mundo académico y empresarial (que demostramos en esta investigación), respecto a que planificando las capacidades requeridas de los recursos en una empresa manufacturera se garantiza que el plan de producción real sea tal como el plan de producción planeado. Sin embargo, eso no basta; es necesario regular el flujo de producción. $\mathrm{Y}$ aun cuando el marco teórico lo advierte, en la práctica no se le da la debida importancia a la regulación del flujo para lograr los objetivos 
planeados, de fechas de entrega, servicio al cliente y costos, entre otros.

Con el Juego propuesto, queremos romper este paradigma, y hacer posible que las personas que se entrenen con este recurso, palpen, reconozcan y se convenzan de todos los problemas originados por no controlar el flujo, y además aprendan cómo deben aplicar el sistema DBR propuesto por el Modelo de Teoría de Restricciones para regular y controlar la tasa de avance de los inventarios en piso de planta.

- Segundo: tanto a nivel de PreGrado como de Post-Grado, en las Escuelas de Administración e Ingeniería el alumno es formado en el estudio de técnicas para gestionar empresas manufactureras o de servicios; $y$, por tanto, es conducido al estudio de uno de los tres Modelos de Referencia para administrar el negocio, el estudio de la Teoría de Restricciones (Theory of Constraints System - TOC).

Los que enseñamos el Modelo de Teoria de Restricciones, sentimos los problemas de transmitir conceptos y principios que de por sí son abstractos y complejos de entender, y que se vuelven más dificiles cuando el estudiante carece de experiencia profesional, o cuando el estudiante maneja conceptos de los otros Modelos de Referencia, los cuales colisionan directamente con el Modelo de Teoría de Restricciones. En este último caso, el alumno tiene que romper una serie de esquemas pre-establecidos para asimilar lo propuesto por el Modelo de Teoría de Restricciones.

La ilustración de los conceptos en el aula se hace utilizando gráficos, esquemas mentales, fórmulas, técnicas de programación finitas e infinitas, videos de empresas, que ha implementado el sistema TOC. Sin embargo, a pesar de toda esta información, el alumno tiene muy poca oportunidad de interactuar con toda la dinámica de ese proceso.

Por tanto, nuestro objetivo principal en el desarrollo de este estudio ha sido demostrar que el Juego Propuesto es efectivamente un recurso capaz de proporcionar un aprendizaje significativamente mayor que otros recursos que se utilicen para enseñar el Planeamiento y Control de la Producción, según el Modelo de Teoría de Restricciones.

\section{DESARROLLO METODOLÓGICO}

\section{Diseño de la Investigación}

Para responder a las preguntas de una investigación, cumplir con los objetivos del estudio, así como someter las hipótesis formuladas a prueba, se desarrolló una investigación experimental.

El uso del Juego Propuesto es nuestra variable independiente, y hemos manipulado intencionalmente esta variable para analizar las conse- 
cuencias que se manifiestan sobre las variables dependientes.

La investigación experimental está compuesta por dos experimentos, y en ambos hemos utilizado un Grupo de Control y un Grupo Experimental. Estos grupos son similares en todo menos en la manipulación de la variable independiente.

Hemos expuesto al Grupo Experimental a la presencia del Juego; mien- tras que el Grupo de Control trabajó utilizando Hoja de Cálculo con números aleatorios. Posteriormente, hemos medido los resultados para saber si los logros en el aprendizaje del planeamiento y control de la producción, según el Modelo de Teoría de restricciones, difieren entre ambos grupos.

Las Figuras 1 y 2 resumen gráficamente los objetivos y diseños de los dos experimentos realizados.

Figura 1. Relación del Primer Objetivo con el Experimento $\mathrm{N}^{\circ} 1$

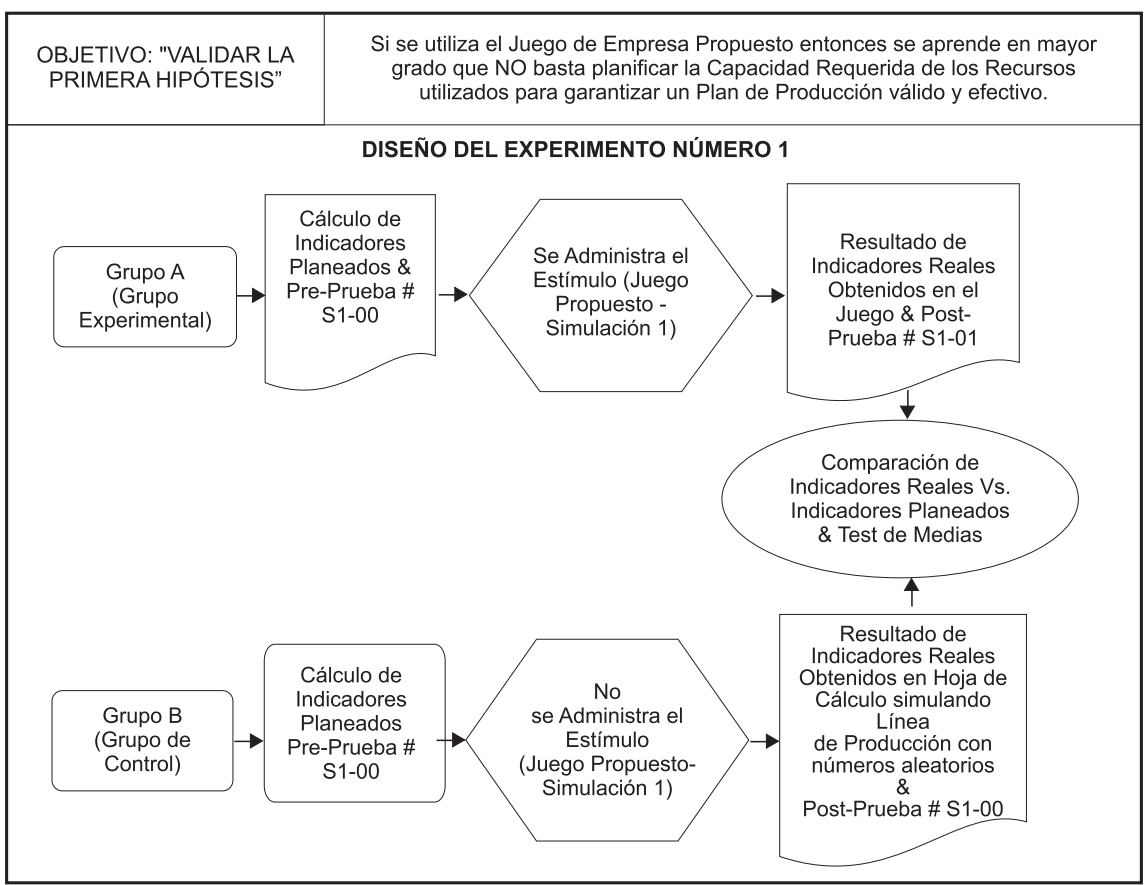


Figura 2. Relación del Segundo y Tercer Objetivo con el Experimento $\mathrm{N}^{\circ} 2$

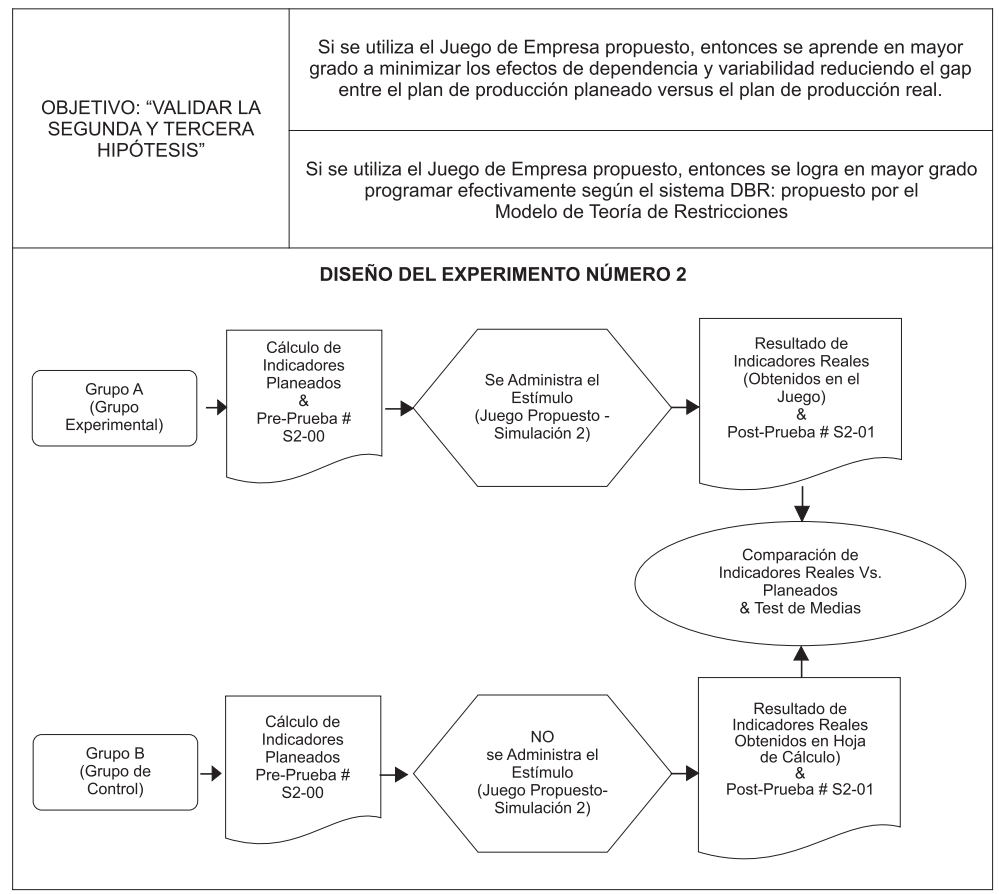

\section{Población y Muestra}

Las muestras no probabilísticas dirigidas estuvieron conformadas por grupos de alumnos del curso de Planeamiento Táctico y Estratégico de Operaciones dictado en la Escuela de Posgrado, Maestría en Ingeniería Industrial, de la Universidad Ricardo Palma (Lima - Perú).

Dado que nuestro interés tiene como objetivo analizar una muestra concreta para verificar si esta innovación didáctica (el uso del juego propuesto) funciona en clase, el tamaño de cada muestra (o cada grupo) fue de ocho alumnos. Este tamaño no es arbitrario, sino más bien está dictado por el propio juego, pues en él se simula una línea de producción con ocho centros de trabajo. Es decir, cada alumno se desempeña como si fuera un operario de una estación de trabajo.

En sintesis, ocho alumnos han sido sometidos a un aprendizaje utilizando el juego propuesto (Grupo Experimental); y otro grupo de ocho alumnos ha simulado la misma Línea de Producción con Hojas de Cálculo (Grupo de Control).

\section{Técnica para Recolección y Proce- samiento de Datos}

En ambos Experimentos las fuentes de datos obtenidas son primarias, debido a que estos se levantaron directamente de los experimentos dise- 
ñados. A su vez, estas fuentes primarias las recogimos de dos maneras:

- Primero: "Por Observación Directa" a Ambos grupos (el experimental y el de control), calcularon y registraron los indicadores reales obtenidos (Eficiencia de la Línea, Eficiencia de cada Centro de Trabajo, Unidades Realmente Producidas, Inven- tario Final en Proceso, y Lead Time de Producción), luego de haber puesto a trabajar la Línea de Producción por 20 dias.

- Segundo: "Por Cuestionario" a Ambos grupos (el experimental y el de control), fueron sometidos a Pre-Pruebas y Post-Pruebas, como se señala en la Tabla 1.

Tabla 1. Aplicación de Cuestionarios al Grupo Experimental y al Grupo de Control.

\begin{tabular}{|c|c|c|c|}
\hline Experimento & Objetivo de la Prueba & $\begin{array}{c}\text { Grupo } \\
\text { Experimental }\end{array}$ & $\begin{array}{c}\text { Grupo } \\
\text { De Control }\end{array}$ \\
\hline \multirow{2}{*}{ 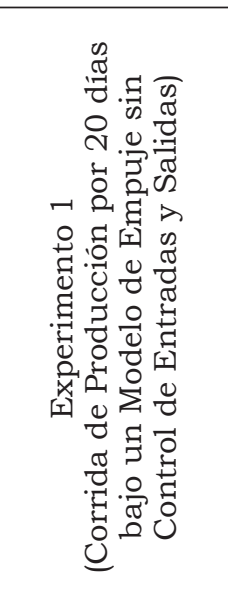 } & $\begin{array}{l}\text { La Pre-Prueba S1-00, es un cues- } \\
\text { tionario cerrado, aplicado antes } \\
\text { de ejecutar el Experimento } 1 \text {, cuyo } \\
\text { objetivo es evaluar cuáles son los } \\
\text { resultados reales que los alumnos } \\
\text { esperan obtener luego de una corri- } \\
\text { da de producción de } 20 \text { días bajo el } \\
\text { modelo de empuje sin control de en- } \\
\text { tradas y salidas. }\end{array}$ & $\begin{array}{l}\text { Pre-Prueba } \\
\text { S1-00 }\end{array}$ & $\begin{array}{l}\text { Pre-Prueba } \\
\text { S1-00 }\end{array}$ \\
\hline & $\begin{array}{l}\text { La Post-Prueba S1-01, es un cues- } \\
\text { tionario abierto, aplicado al finalizar } \\
\text { el Experimento } 1 \text {, cuyo objetivo es } \\
\text { evaluar si los alumnos han detecta- } \\
\text { do cuáles son las causas raíz de por- } \\
\text { qué la Línea de Producción luego de } \\
\text { la corrida de } 20 \text { días, no se ha des- } \\
\text { empeñado de acuerdo a lo planeado. }\end{array}$ & $\begin{array}{l}\text { Post-Prueba } \\
\text { S1-01 }\end{array}$ & $\begin{array}{l}\text { Post-Prueba } \\
\text { S1-01 }\end{array}$ \\
\hline \multirow{2}{*}{ 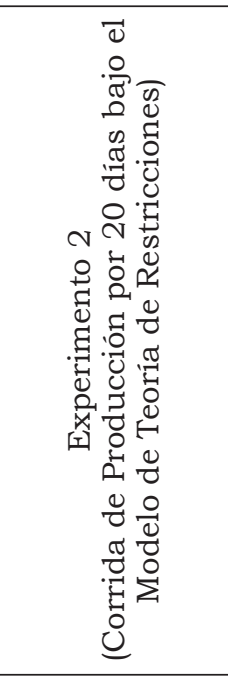 } & $\begin{array}{l}\text { La Pre-Prueba S2-00, es un cuestio- } \\
\text { nario abierto, que evalúa cómo los } \\
\text { alumnos piensan planear la Linea } \\
\text { de Producción bajo un Sistema de } \\
\text { Teoría de Restricciones. Ellos de- } \\
\text { ben identificar el tambor, la cuerda, } \\
\text { calcular los buffers de protección y } \\
\text { de ensamble, el inventario inicial en } \\
\text { proceso, así como definir la métrica } \\
\text { que van a utilizar (eficiencia, utiliza- } \\
\text { ción, productividad) y a qué centros } \\
\text { de trabajo le van a exigir esa métrica } \\
\text { para lograr los objetivos planeados }\end{array}$ & $\begin{array}{l}\text { Pre-Prueba } \\
\text { S2-00 }\end{array}$ & $\begin{array}{l}\text { Pre-Prueba } \\
\quad \text { S2-00 }\end{array}$ \\
\hline & $\begin{array}{l}\text { La Post-Prueba S2-01, es un cues- } \\
\text { tionario abierto, donde se registra } \\
\text { los resultados reales de la Linea de } \\
\text { Producción, luego de una corrida de } \\
20 \text { días bajo el Modelo de Teoría de } \\
\text { Restricciones. }\end{array}$ & $\begin{array}{l}\text { Post-Prueba } \\
\quad \text { S2-01 }\end{array}$ & $\begin{array}{l}\text { Post-Prueba } \\
\text { S2-01 }\end{array}$ \\
\hline
\end{tabular}


Los resultados de las Pre-Pruebas y Post-Pruebas de ambos experimentos fueron la base de datos para comprobar si las diferencias observadas en términos de aprendizaje son significativas entre el Grupo Experimental y el de Control.

Asimismo, al concluir ambos experimentos se aplicó tanto al Grupo Experimental como al Grupo de Control los Cuestionarios ER-00 \& ER-01, para evaluar cuál de las dos metodo- logías (el Juego o la Hoja de Cálculo) es más apreciada y valorada por los estudiantes.

\section{RESULTADOS}

\section{Relacionados con el Primer Experi- mento}

En la Tabla 2, se resumen los resultados de la estadística descriptiva e inferencial, que están relacionados con el Primer Experimento.

Tabla 2. Resumen de Resultados del experimento $\mathrm{N}^{\circ} 1$

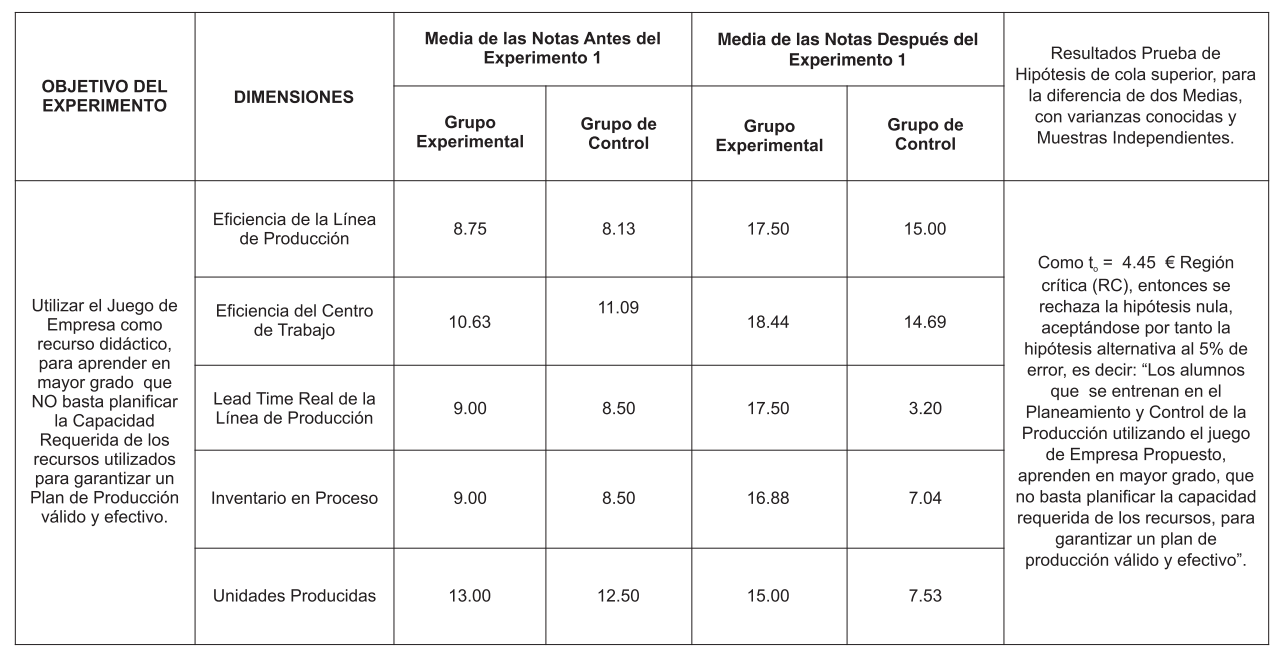

Nota:

- Las Medias de las Notas antes del Experimento $\mathrm{N}^{\circ} 1$ se recogen en la Pre-Prueba S1-00

- Las Medias de las Notas después del Experimento $N^{\circ} 1$ se recogen en la Pre-Prueba 1 1-01

- Todas las Medias de las Notas están en una Escala Vigesimal

- Mediante una Prueba de Hipótesis de cola superior, para la diferencia de dos Medias, con Varianzas conocidas y Muestras Independientes, quedó demostrado con un error del 5\% y un $\mathrm{t}_{0}=4.45 \in \mathrm{RC}$, que los alumnos que se entrenan en el Planeamiento y Control de la Producción utilizando el Juego de Empresa Propuesto, aprenden en mayor grado que no basta planificar la capacidad requerida de los recursos para garantizar 
un plan de producción válido y efectivo

- Es pertinente subrayar que el Cuestionario Abierto S1-01, aplicado a ambos grupos al finalizar el Experimento $\mathrm{N}^{0} 1$, lo que busca es detectar si los alumnos logran identificar las causas raíz de por qué la Línea de Producción no ha alcanzado los resultados planeados, luego de una corrida de veinte dias bajo un sistema de empuje sin control de entradas-salidas.

En función a los resultados obtenidos, se evidenció que los alumnos que utilizaron el Juego Propuesto consiguieron identificar que la falta de regulación del flujo productivo era la principal causa raíz del mal desempeño de la línea. Consideramos que esto fue posible porque a lo largo de todo el juego, ellos pudieron ver efectos concretos que se manifestaban como resultado de la desregulación del flujo, como por ejemplo: dilatación de colas, incremento del work in process, demora de los inven- tarios en atravesar la línea de producción, entre otros; y consiguieron también comprender mejor conceptos más teóricos como el fenómeno de la dependencia y variabilidad.

Para los alumnos que trabajaron con números aleatorios les resultó más difícil analizar la información numérica obtenida. Aunque contaban con más información en tiempo real, les era más complejo encontrar las causas raíz de los problemas; y los resultados de las pruebas así lo demuestran.

Por tanto, esto corroboraría lo sostenido por Freitas y Santos [2005], en el sentido de que los Juegos de Empresas son herramientas que facilitan mejor el proceso de aprendizaje, contribuyendo a mejorar la comprensión de asuntos abstractos

\section{Relacionados con el Segundo Expe- rimento}

La Tabla 3 sintetiza los resultados relacionados con el Segundo Experimento propuesto en esta investigación. 
Tabla 3. Resumen de Resultados del Experimento $\mathrm{N}^{\circ} 2$

\begin{tabular}{|c|c|c|c|c|c|}
\hline \multirow{2}{*}{$\begin{array}{c}\text { PRIMER } \\
\text { OBJETIVO DEL } \\
\text { EXPERIMENTO } \\
\mathrm{N}^{\circ} 2\end{array}$} & \multirow{2}{*}{\multicolumn{2}{|c|}{ DIMENSIONES }} & \multicolumn{2}{|c|}{ Post Prueba S2-01 } & \multirow{2}{*}{$\begin{array}{l}\text { Resultados Prueba de } \\
\text { Hipótesis de cola superior, } \\
\text { para la diferencia de dos } \\
\text { Medias, con Varianzas } \\
\text { diferente y Muestras } \\
\text { Independientes }\end{array}$} \\
\hline & & & $\begin{array}{c}\text { Grupo } \\
\text { Experimental }\end{array}$ & $\begin{array}{c}\text { Grupo de } \\
\text { Control }\end{array}$ & \\
\hline \multirow{9}{*}{$\begin{array}{c}\text { Utilizar el Juego } \\
\text { de Empresa } \\
\text { como recurso } \\
\text { didáctico, para } \\
\text { aprender en } \\
\text { mayor grado a } \\
\text { minimizar los } \\
\text { efectos de } \\
\text { Dependencia y } \\
\text { Variabilidad, } \\
\text { reduciendo el } \\
\text { gap entre el } \\
\text { Plan de } \\
\text { Producción } \\
\text { Planeado versus } \\
\text { el Plan de } \\
\text { Producción } \\
\text { Real. }\end{array}$} & \multirow{9}{*}{$\begin{array}{l}\text { Eficiencia del } \\
\text { Sistema de } \\
\text { Producción }\end{array}$} & Eficiencia Real CT1 & $80 \%$ & $77 \%$ & \multirow{9}{*}{$\begin{array}{l}\text { Como tq }=4.23 \text { pertenece a la } \\
\text { Región Crítica (RC), entonces } \\
\text { se rechaza la hipótesis nula, } \\
\text { aceptándose la hipótesis } \\
\text { alternativa, es decir: "Los } \\
\text { alumnos que se entrenan en } \\
\text { el Planeamiento y Control de } \\
\text { la Producción utilizando el } \\
\text { Juego de Empresa Propuesto, } \\
\text { aprenden en mayor grado, a } \\
\text { minimizar los efectos de } \\
\text { dependencia y variabilidad } \\
\text { reduciendo el gap entre el } \\
\text { plan de producción planeado y } \\
\text { el plan de producción real". }\end{array}$} \\
\hline & & Eficiencia Real CT2 & $80 \%$ & $77 \%$ & \\
\hline & & Eficiencia Real CT3 & $80 \%$ & $77 \%$ & \\
\hline & & Eficiencia Real CT4 & $80 \%$ & $77 \%$ & \\
\hline & & Eficiencia Real CT5 & $80 \%$ & $79 \%$ & \\
\hline & & Eficiencia Real CT6 & $80 \%$ & $76 \%$ & \\
\hline & & Eficiencia Real CT7 & $83 \%$ & $73 \%$ & \\
\hline & & Eficiencia Real CT8 & $81 \%$ & $70 \%$ & \\
\hline & & Eficiencia Real de la Línea & $95 \%$ & $85 \%$ & \\
\hline
\end{tabular}

Nota:

- Los Resultados de la Post-Prueba S2-01, registran las Eficiencias Reales logradas por cada Grupo al Finalizar el Experimento №2

- Las Eficiencias Reales están dadas en términos porcentuales

- Compare el Resultado Real de la Eficiencia de la Línea con el Resultado Planeado que era de

- Compare los Resultados Reales de la Eficiencia de cada Centro de Trabajo con el Planeado que

- Con el Experimento $\mathrm{N}^{\circ} 2$ se pudo verificar que los alumnos que utilizaron el Juego Propuesto exhiben un conspicuo aprendizaje de la Teoría de Restricciones, porque lograron eficiencias reales que se aproximan más a las eficiencias planeadas, con lo cual acreditaron una buena programación y control de la Linea de Producción. Podemos apreciar en la Tabla 2, que la Eficiencia Real, tanto de la Línea de Producción cuanto las Eficiencias Reales de los ocho centros de trabajo fueron superiores a lo alcanzado por el Grupo de Control.

- Asimismo, mediante un Test de Fischer se comprobó las varian- zas de las notas obtenidas en la Post Prueba, tanto del Grupo Experimental como del Grupo de Control, y procedimos a hacer una Prueba de Hipótesis de cola superior, para la diferencia de dos Medias, con Varianzas Diferentes y Muestras Independientes; lo cual nos permite aseverar con un error de 5\% que los alumnos que se entrenan en el Planeamiento y Control de la Producción utilizando el Juego de Empresa propuesto, aprenden en mayor grado, a minimizar los efectos de dependencia y variabilidad, reduciendo el gap entre el plan de producción planeado y el plan de producción real. 
La Tabla 4 sintetiza los resultados del Experimento $\mathrm{N}^{\circ} 2$. relacionados con el Segundo Objetivo

Tabla 4. Resumen de Resultados del Experimento ${ }^{\circ} 2$

\begin{tabular}{|c|c|c|c|c|c|}
\hline \multirow{2}{*}{$\begin{array}{l}\text { SEGUNDO OBJETIVO } \\
\text { DEL EXPERIMENTO N²2 }\end{array}$} & \multirow{2}{*}{\multicolumn{2}{|c|}{ DIMENSIONES }} & \multicolumn{2}{|c|}{ Post Prueba S2-01 } & \multirow{2}{*}{$\begin{array}{l}\text { Resultados Prueba de Hipótesis de } \\
\text { cola superior, para la diferencia de } \\
\text { dos Medias, con Varianzas diferentes } \\
\text { y Muestras Independientes. }\end{array}$} \\
\hline & & & Grupo & Grupo de & \\
\hline \multirow{3}{*}{$\begin{array}{l}\text { Utilizar el Juego de } \\
\text { Empresa como recurso } \\
\text { didáctico, para aprender } \\
\text { en mayor grado a } \\
\text { programar efectivamente } \\
\text { según el sistema DBR: } \\
\text { (Drum-Burfer-Fope) } \\
\text { propuesto por el }\end{array}$} & \multirow{3}{*}{$\begin{array}{l}\text { Sistema de } \\
\text { Programación } \\
\text { DBR. }\end{array}$} & $\begin{array}{c}\text { Unidades Realmente } \\
\text { Manufacturadas por la } \\
\text { Línea de Producción } \\
\text { (en unidades) }\end{array}$ & 57 & 51 & \multirow{3}{*}{$\begin{array}{c}\text { Como tq }=3.17 \text { pertenece a la Región } \\
\text { Crítica (RC), entonces se rechaza la } \\
\text { hipótesis nula, aceptandose por tanto } \\
\text { la hipótesis alternativa al } 5 \% \text { de error, } \\
\text { es decir: "Los alumnos que se } \\
\text { entrenan en el Planeamiento y } \\
\text { Control de la Producción utilizando el } \\
\text { Juego de Empresa Propuesto } \\
\text { aprenden en mayor grado, a } \\
\text { programar efectivamente según el } \\
\text { sistema DBR: propuesto por el } \\
\text { Modelo de Teoría de Restricciones" }\end{array}$} \\
\hline & & $\begin{array}{l}\text { Inventario en Proceso } \\
\text { (en unidades) }\end{array}$ & 37 & 46 & \\
\hline & & $\begin{array}{l}\text { Lead Time Real de } \\
\text { Producción } \\
\text { (en días) }\end{array}$ & 12 & 14 & \\
\hline
\end{tabular}

Nota:

- Los Resultados de la Post-Prueba S2-01, registran los Resultados Reales logrados por cada Grupo al Finalizar el Experimento N 2

- Compare los Resultados Reales de Producción de la Línea contenidos por el Grupo Experimental y de Control con el Resultado Planeado que era de 60

- Compare los Resultados Reales de Inventarlo en Proceso Finales de la Línea contenidos por el Grupo Experimental y de Control, con el inventario inicial que era de 24 unidades.

- Compare los Resultados Reales del Lead Time de Producción obtenidos por el Grupo Experimental y de Control, con el Lead Time Planeado de la Línea que era de 7 días.

- Con el Experimento $\mathrm{N}^{\circ} 2$ se pudo cotejar que los alumnos que utilizaron el Juego Propuesto, aplicaron significativamente mejor el mecanismo DBR propio del Modelo de Teoría de Restricciones para planear y programar la Línea de Producción. Los resultados de la Tabla 4 muestran con claridad que luego de una corrida de producción de veinte dias, el Grupo Experimental manufactura 57 unidades, versus las 51 fabricadas por el Grupo de Control. Asimismo, el Tiempo Real que le demora a la materia prima convertirse en producto terminado (Lead Time Real de Producción), es de 12 días para el Grupo Experimen- tal y de 14 días para el Grupo de Control. Finalmente, el Inventario en Proceso al Finalizar los veinte dias de producción, es de 57 unidades para el Grupo Experimental, versus 51 unidades para el Grupo de Control.

- Asimismo, mediante la PrePrueba S2-00, la que se aplica antes de iniciar el Experimento $\mathrm{N}^{\circ} 2$, pudimos constatar que en el Grupo Experimental, seis de ocho alumnos identificaron bien los Centros de Trabajo que conforman la Cuerda, mientras que en el Grupo de Control, solo 1 de 8 alumnos logró hacer lo mismo.

- Esto significa que el concepto de CUERDA fue mucho mejor 
comprendido y trabajado por el Grupo Experimental que por el Grupo de Control.

En el Grupo Experimental, el $37.5 \%$ de los alumnos dimensionó muy bien los buffers, y un $50 \%$ lo hizo bien. Por otro lado, en el Grupo de Control solo el $37.5 \%$ de los alumnos dimensionó bien los buffers, mientras que el $62.5 \%$ lo hizo muy mal. Esto significa que el Grupo Experimental cuantificó, localizó y aplicó mucho mejor el concepto del Buffer que el Grupo de Control. Respecto al DRUM, el 100\% de los alumnos del Grupo Experimental manifiestan correctamente cuáles son los Centros de Trabajo que deben fabricar lo que manufacture el cuello de botella. Sin embargo, solo el $50 \%$ de los alumnos del Grupo de Control han logrado entender que los Centros de Trabajo que no trabajen a sus propias capacidades deben subordinarse al cuello de botella.

Finalmente, mediante prueba de hipótesis de cola superior, para la diferencia de dos medias, con varianzas diferentes y muestras independientes, concluimos con $5 \%$ de error que los alumnos que se entrenan en el Planeamiento y Control de la Producción utilizando el Juego de Empresa Propuesto, aprenden en mayor grado a programar efectivamente según el sistema DBR propuesto por el Modelo de Teoría de Restricciones.

Tabla 5. Resultados Agregados de la Valoración de los Recursos Utilizados

\begin{tabular}{|c|c|c|c|c|c|}
\hline \multirow{2}{*}{$\begin{array}{l}\text { Pregunta } \\
\mathrm{N}^{0}\end{array}$} & \multirow{2}{*}{ Indicador } & \multicolumn{2}{|c|}{$\begin{array}{c}\text { Grupo Experimental } \\
\text { (Utilizó el Juego Propuesto) }\end{array}$} & \multicolumn{2}{|c|}{$\begin{array}{c}\text { Grupo de Control } \\
\text { (Utilizó Números Aleatorios) }\end{array}$} \\
\hline & & Promedio & $\begin{array}{c}\text { Desviación } \\
\text { Estándar }\end{array}$ & Promedio & $\begin{array}{c}\text { Desviación } \\
\text { Estándar }\end{array}$ \\
\hline 1 & Refuerza / Aprueba & 4.0000 & 0.0000 & 2.5000 & 0.5345 \\
\hline 2 & \multirow{5}{*}{ Valora } & 4.0000 & 0.0000 & 2.5000 & 0.5345 \\
\hline 3 & & 3.6250 & 0.5175 & 3.1250 & 0.6409 \\
\hline 4 & & 3.7500 & 0.4629 & 2.7500 & 0.4629 \\
\hline 5 & & 3.5000 & 0.5345 & 3.2500 & 0.4629 \\
\hline 6 & & 3.7500 & 0.4629 & 2.3750 & 0.5175 \\
\hline 7 & \multirow{2}{*}{ Motivación } & 4.0000 & 0.0000 & 2.5000 & 0.5345 \\
\hline 8 & & 4.0000 & 0.0000 & 2.6250 & 0.5175 \\
\hline
\end{tabular}

Nota:

- Los Resultados del Grupo Experimental se recogieron mediante el instrumento ER-00.

- Los Resultados del Grupo de Control se recogieron mediante el instrumento ER-01.

- Las Medias de las notas están en una escala de Likert que va de CERO a CUATRO 
Relacionados con la Valoración del Recurso Utilizado

- En la Tabla 5, advertimos que todos los indicadores utilizados para medir la opinión crítica de los alumnos respecto a los recursos empleados se inclinaron rotundamente al Juego propuesto.

- También se hizo una prueba de hipótesis de cola superior para la diferencia de dos medias, con varianzas conocidas y muestras independientes, y se concluyó con $5 \%$ de error, que los alumnos valoran más el recurso Juego de Empresa cuando se entrenan para aprender a Planear y Controlar la Producción según el Modelo de Teoría de Restricciones.

- En este trabajo, se recogen datos que permitieron obtener otros resultados. Así pues, mediante el Cuestionario S1-00, previo a la ejecución del Experimento $\mathrm{N}^{\circ} 1$ realizado en este trabajo, al ser preguntados los alumnos cuáles esperan que sean los resultados reales que logre la Línea de Producción luego de una corrida de veinte dias bajo un sistema de empuje sin control de entradas y salidas, se evidencia que todos los estudiantes (tanto los del Grupo Experimental como los del Grupo de Control), responden que esperan que los resultados reales sean tal como lo planeado.

Esto corroboraria el hecho del paradigma que arrastran las personas, respecto a que consideran que planificando bien la capacidad o los recursos necesarios, los resultados reales de producción están garantizados. Los alumnos tienen ante sí una línea de producción casi perfectamente bien balanceada en términos de recursos, y suponen (erradamente) que está garantizado que los resultados reales de la Línea sean iguales a lo planeado. Es decir, olvidan lo que sustenta el marco teórico, en el sentido que no importa cuál sea el Modelo de Referencia que utilicemos para planear inventarios y recursos en una empresa; todos los Modelos exigen mecanismos de control del flujo de producción, pues de lo contrario todos los esfuerzos de planificación se verán diluidos y será imposible lograr el desempeño planeado del sistema productivo.

- A pesar que los alumnos han sido entrenados en los conceptos de Teoría de Restricciones, cuando tienen frente a sí una Línea muy bien balanceada, asumen que los resultados reales serán tan iguales que los planeados.

En esta investigación, se hicieron tres Pruebas de Proporciones para demostrar si efectivamente este paradigma se manifiesta en ambos grupos: el experimental y el de control. 
En la primera Prueba de Proporciones, se demuestra que no hay diferencia significativa entre las proporciones de los alumnos que esperan que habiendo planeado la Capacidad de los Recursos, la Eficiencia Real sea igual a la Eficiencia Planeada de la Línea de Producción, al 5\% de error. ciones. Por ello, nos permitimos recomendar su uso en ambientes académicos, tanto a nivel de pregrado como de postgrado, donde se impartan cursos de Teoría de Restricciones en particular.

- La aplicación del juego propuesto en esta investigación contribuirá a comprender la importancia crítica que tiene la regulación del flujo de producción en los ambientes manufactureros. El Juego contribuirá, asimismo, a practicar, experimentar y aprender cómo se hace el control del flujo.

\section{RECOMENDACIONES}

La tercera Prueba de Hipótesis confirma que tampoco hay diferencia entre las proporciones de los alumnos que esperan que habiendo planeado la Capacidad de los Recursos, el Lead Time Real sea igual al Lead Time Planeado de la Línea de Producción, con $5 \%$ de error.

\section{CONCLUSIONES}

- Hemos demostrado en este trabajo que el uso del Juego propuesto, no solo ha gustado, motivado y logrado amplia aprobación de los alumnos que lo utilizaron, sino principalmente que ha sido un mecanismo muy efectivo en el aprendizaje del Modelo de Teoría de Restric-
- El Juego aplica para cursos de Gestión de Operaciones, Planeamiento y Control de la Producción, Administración Logística, entre otros, pues todos ellos planifican, programan y controlan inventarios y recursos, procesos que se simulan en el Juego propuesto.

- Aconsejamos este recurso, porque se aprende mejor haciendo. $\mathrm{Y}$, como hemos demostrado, aún conceptos difusos y complejos como los de Teoria de Restricciones, han sido aprendidos y asimilados con más facilidad por los alumnos que utilizaron esta herramienta. 


\section{REFERENCIAS BIBLIOGRÁFICAS}

Almeida F.C. (1998). Experiências no uso de jogos de empresas no ensino de Administração. In. Seminarios de Administração 3., São Paulo. Anais. São Paulo: FEA/USP.

Bastos-De Figueiredo J. (2010). Modelo Computacional para Simulação de Aplicação da Teoria das Restrições. Tesis para obtener el Grado de Doctor en Administración de la Producción y de Operaciones. Universidad de São Paulo. São Paulo, Marzo 2010.

Bernard R. (2006). Métodos de Jogos de Empresa/Simulação Gerencial”. In: MARION, José Carlos; MARION, Arnaldo Luis Costa. Metodologias de Ensino na Área de Negócios, São Paulo: Atlas. p. 83-114.

Ingo Lange, A.Z. (2005). New Approaches on Learning, Studying and Teaching - The Constraints Game - Learning the Theory of Constraints with a Dice Game. In. 9th International Workshop on Experimental Interactive Learning, Studying and Teaching. Swiss Federal Institute of Technology ETH Zurich. Finland.

Kolb D.A. (1997). A Gestão e o Processo de Aprendizagem, In. Starkey K. Como as organizações aprendem: relatos do sucesso das grandes empresas. Ed. Futura, São Paulo.

Lane D. (1995). On a resurgence of management simulations and Games. Journal of the operational Research Society. London School of Economics and Political Science. Londres.

Lopez P.C. (2001). Jogos de Empresas Geral: a perspectiva do animador com a utilização na pós-graduação lato sensu. In. Encontro Anual da Associação dos Programas de Pós-graduação em Administração, 25, Campinas, EPA-136, CD-ROM.

Martinelli D.P. (1998). A utilização dos Jogos de Empresas no Ensino de Administração. In. Revista de Administração, v.23, n.3, p.24-37, São Paulo: jul./ set. 1998.

Sauaia, A.C. (2006). Gestão Empreendedora em IES`s: Aculturamento do Corpo Docente com Jogos de Empresas. In. Colóquio Internacional sobre Gestão Universitária na América do Sul. VI, Blumenau.

Seaton L. J.; Boyd M. (2008). The Effective Use Of Simulations In Business Courses. Academy of Educational Leadership Journal, v. 12, n. 1, 2008.

Souza P.R.; Lopez P.C. (2004). Jogos de Negócios como Ferramentas para a Construção de Competências Essenciais às Organizações. In. Seminarios de Administração. 7., USP, São Paulo. 